Revista de Matemática: Teoría y Aplicaciones 2007 14(1) : 81-100

CIMPA - UCR - CCSS ISSN: 1409-2433

\title{
DIFFERENTIALLY CLOSED FIELDS OF CHARACTERISTIC ZERO WITH A GENERIC AUTOMORPHISM
}

\author{
Ronald F. Bustamante Medina* \\ Recibido/Received: 18 Oct 06 - Aceptado/Accepted: 31 Jan 2007
}

\begin{abstract}
Hrushovski showed that the theory of difference-differential fields of characteristic zero has a model-companion, which we shall denote $D C F A$. We give an axiomatization for $D C F A$ and prove some important model-theoretic results as supersimplicity and elimination of imaginaries. We mention some properties of the fixed field and the constant field of a model of DCFA.
\end{abstract}

Keywords: Mathematical logic, model theory, differential fields, difference fields

\section{Resumen}

Hrushovski demostró que la teorìa de campos diferenciales de diferencia de característica cero tiene una modelo-compaẽra, la cual denotaremos $D C F A$. En el presente artículo damos una axiomatización para $D C F A$ y probamos algunos resultados modeloteoréticos de importancia como la supersimplicidad y la eliminación los imaginarios. Mencionamos algunas propiedades del campo fijo y del campo de constantes de un modelo de $D C F A$.

Palabras clave: Lógica matemática, teoría de modelos, campos diferenciales, campos de diferencia.

Mathematics Subject Classification: 11U09, 12H05, $12 \mathrm{H} 10$.

\section{Introduction}

A difference-differential field is a differential field with an automorphism which commutes with the derivation.

\footnotetext{
*CIMPA, Escuela de Matemática, Universidad de Costa Rica, 2060 San José, Costa Rica. E-Mail: bustam@gmail.com
} 
E. Hrushovski proved that, in characteristic zero, the theory of difference-differential fields has a model-companion.

In this work we give a proof of Hrushovski's theorem: we give an explicit axiom scheme for the model-companion of the theory of difference-differential fields of characteristic zero. This theory is called DCFA.

Next we give a description of the algebraic closure, types, and completions of $D C F A$, we define an independence relation and we mention some properties of $D C F A$ as supersimplicity and elimination of imaginaries. We also give some results concerning the fixed field and the field of constants of a model of DCFA.

\section{Differential Fields and Difference Fields}

First we mention some results about differential algebra and the theory of differentially closed fields. Even if some of the results hold for all characteristics we shall work in fields of characteristic 0 .

As a convention we will assume that all varieties are absolutely irreducible.

We assume that the reader is acquainted with the basic properties in differential algebra and differentially closed fields. For results in differential algebra we refer to [7], for modeltheoretic results see [10], [11], [12] and [16].

Let $(K, D)$ be a differential field, and let $K[X]_{D}$ denote the ring of differential polynomials in $X=\left(X_{1}, \cdots, X_{n}\right)$. Let $f \in K[X]_{D}$. The differential order of $f$, denoted $\operatorname{ord}_{D}(f)$, is the greatest integer $n$ such that $D^{n} X$ appears in $f$ with non-zero coefficient. If there is no such $n$ we set $\operatorname{ord}_{D}(f)=-1$.

Let $I$ be an ideal of $K[X]_{D}$. We say that $I$ is a differential ideal if it is closed under $D$. If $I$ is a differential ideal of $K[X]_{D}$, then $K[X] / I$ is a differential ring.

Proposition 2.1 If $(K, D)$ is a differential field, then $K\left[X_{1}, \cdots, X_{n}\right]_{D}$ satisfies the ascending chain condition on radical differential ideals.

We define the $D$-topology of $K^{n}$ (also called Kolchin topology or Zariski differential topology), as the topology with the sets of the form $V_{D}(I)=\left\{x \in K^{n}: f(x)=0 \quad \forall f \in I\right\}$ as basic closed sets, where $I$ is a differential ideal of $K\left[X_{1}, \cdots, X_{n}\right]_{D}$. From 2.1 we deduce that the $D$-topology of $K^{n}$ is Noetherian.

An important result in differential algebraic geometry is Kolchin's Irreducibility Theorem, see e.g. [10], Chapter II, Appendix C.

Proposition 2.2 Let $(K, D)$ be a differential field, and let $V$ be an algebraic variety defined over $K$. Then $V$ is irreducible in the D-topology.

Definition 2.3 Let $(K, D)$ be a differential field, and let $V \subset \mathbb{A}^{n}$ be a variety, let $F(X)$ be a finite tuple of polynomials over $K$ generating $I(V)$ where $X=\left(X_{1}, \cdots, X_{n}\right)$.

1. We define the first prolongation of $V, \tau_{1}(V)$ by the equations:

$$
F(X)=0, J_{F}(X) Y_{1}^{t}+F^{D}(X)=0
$$


where $Y_{1}$ is an n-tuple, $F^{D}$ denotes the tuple of polynomials obtained by applying $D$ to the coefficients of each polynomial of $F$, and $J_{F}(X)$ is the Jacobian matrix of $F$ (i.e. if $F=\left(F_{1}, \cdots, F_{k}\right)$ then $\left.J_{F}(X)=\left(\partial F_{i} / \partial X_{j}\right)_{1 \leq i \leq k, 1 \leq j \leq n}\right)$.

2. For $m>1$, we define the $m$-th prolongation of $V$ by induction on $m$ :

Assume that $\tau_{m-1}(V)$ is defined by $F(X)=0, J_{F}(X) Y_{1}^{t}+F^{D}(X)=0, \cdots$, $J_{F}(X) Y_{m-1}^{t}+f_{m-1}\left(X, Y_{1}, \cdots, Y_{m-2}\right)=0$. Then $\tau_{m}(V)$ is defined by:

$$
\left(X, Y_{1}, \cdots, Y_{m-1}\right) \in \tau_{m-1}(V)
$$

and

$$
\begin{gathered}
J_{F}(X) Y_{m}^{t}+J_{F}^{D}(X) Y_{m-1}^{t}+J_{f_{m-1}}\left(X, Y_{1}, \cdots, Y_{m-2}\right)\left(Y_{1}, \cdots, Y_{m-1}\right)^{t} \\
+f_{m-1}^{D}\left(X, Y_{1}, \cdots, Y_{m-2}\right)=0
\end{gathered}
$$

3. Let $W \subset \tau_{m}(V)$ be a variety. We say that $W$ is in normal form if, for every $i \in\{0, \cdots, m-1\}$, whenever $G\left(X, Y_{1}, \cdots, Y_{i}\right) \in I(W) \cap K\left[X, Y_{1}, \cdots, Y_{i}\right]$ then

$$
J_{G}\left(X, Y_{1}, \cdots, Y_{i}\right)\left(Y_{1}, \cdots, Y_{i+1}\right)^{t}+G^{D}\left(X, Y_{1}, \cdots, Y_{i}\right) \in I(W) .
$$

4. Let $W \subset \tau_{m}(V)$ be a variety in normal form.

$A$ point a (in some extension of $K$ ) is an $(m, D)$-generic of $W$ over $K$ if $\left(a, D a, \cdots, D^{m} a\right)$ is a generic of $W$ over $K$ and for $i>m$, we have

$$
\operatorname{tr} . d g\left(D^{i} a / K\left(a, \cdots, D^{i-1} a\right)\right)=\operatorname{tr} . d g\left(D^{m} a / K\left(a, \cdots, D^{m-1} a\right)\right) .
$$

We give now an axiomatization for the theory of differentially closed fields $D C F$ of characteristic zero due to Pierce-Pillay ([12]).

Theorem 2.4 Let $(K, D)$ be a differential field. $K$ is differentially closed if and only if $K$ is algebraically closed and for every variety $V$, if $W$ is an irreducible algebraic subvariety of $\tau_{1}(V)$, such that the projection of $W$ onto $V$ is dominant, then there is a $\in V(K)$ such that $(a, D a) \in W$.

The theory of differentially closed fields is complete and $\omega$-stable; it eliminates quantifiers and imaginaries (see [11]).

Now we mention some properties about the prolongations and varieties in normal form.

Remark 2.5 1. There is a natural projection from $\tau_{m}(V)$ onto $\tau_{m-1}(V)$.

2. The map $\rho: \tau_{m+1}(V) \rightarrow \tau_{1}\left(\tau_{m}(V)\right)$ defined by

$$
\left(x, u_{1}, \cdots, u_{m}\right) \mapsto\left(\left(x, u_{1}, \cdots, u_{m-1}\right),\left(u_{1}, \cdots, u_{m}\right)\right)
$$

defines an isomorphism between $\tau_{m+1}(V)$ and a Zariski-closed subset of $\tau_{1}\left(\tau_{m}(V)\right)$.

The following lemma ([8], chapter $\mathrm{X})$, gives us a condition to extend the derivation of a differential field. 
Lemma 2.6 Let $(K, D)$ be a differential field and $\bar{a}=\left(a_{i}\right)_{i \in I}$ a (possibly infinite) tuple in some extension of $K$. Let $\left\{F_{j}: j \in J\right\}$ be a set of generators of the ideal $I(\bar{a} / K) \subset$ $K\left[X_{i}: i \in I\right]$.

Let $\left(b_{i}\right)_{i \in I}$ be a tuple of $K(\bar{a})$ such that, for all $j \in J$

$$
\sum_{i \in I} \frac{\partial F_{j}}{\partial X_{i}}(\bar{a}) b_{i}+F_{j}^{D}(\bar{a})=0 .
$$

Then $D$ extends to a unique derivation $D^{*}$ on $K\left(a_{i}\right)_{i \in I}$, such that $D^{*} a_{i}=b_{i}$ for all $i \in I$.

Lemma 2.7 Let $D: K \rightarrow \mathcal{U}$ be such that for all $a, b \in K, D(a+b)=D a+D b(*)$ and $D(a b)=a D b+b D a(* *)$. Let $a \in \mathcal{U}$.

1. If a is transcendental over $K$, and $b \in \mathcal{U}$, then there is $D_{1}: K(a) \rightarrow \mathcal{U}$ extending $D$ and satisfying $(*)$ and $(* *)$ such that $D_{1} a=b$.

2. If a is algebraic over $K$, then there is a unique extension $D_{1}$ of $D$ to $K(a)$ satisfying $(*)$ and $(* *)$.

Proof:

(1) For $f(a) \in K(a)$, set $D_{1}(f(a))=f^{\prime}(a) b+f^{D}(a)$. Since $a$ is transcendental over $K$, one checks easily that $(*)$ and $(* *)$ hold.

(2) Let $f(X)=\sum_{i=0}^{n} a_{i} X^{i}$ be the monic minimal polynomial of $a$ over $K$. We define $D_{1} a=-f^{\prime}(a)^{-1} f^{D}(a)$. Every element of $K(a)$ can be written $\sum_{i=0}^{n-1} b_{i} a^{i}$ where the $b_{i}$ are in $K$. We then set

$$
D_{1}\left(\sum_{i=0}^{n-1} b_{i} a^{i}\right)=-\sum_{i=0}^{n-1}\left(D\left(b_{i}\right) a^{i}+i b_{i} D_{1}(a) a^{i-1}\right) .
$$

Clearly, $D_{1}$ satisfies $(*)$, and to check that is satisfies $(* *)$, it suffices to show that $D_{1}\left(a^{n}\right)=$ $n D_{1}(a) a^{n-1}$. Since $a^{n}=-\sum_{i=0}^{n-1} a_{i} a^{i}$, we have

$$
\begin{gathered}
D_{1}\left(a^{n}\right)=-\sum_{i=0}^{n-1}\left(D\left(a_{i}\right) a^{i}+i a_{i} D_{1}(a) a^{i-1}\right) \\
=-f^{D}(a)-\left(f^{\prime}(a)-n a^{n-1}\right) D_{1}(a) \\
=n a^{n-1} D_{1}(a) .
\end{gathered}
$$

Proposition 2.8 Let $(\mathcal{U}, D)$ be a saturated model of $D C F$, let $K=\operatorname{acl}(K) \subset \mathcal{U}$, let $V$ a smooth variety, and $W$ a subvariety of $\tau_{m}(V)$ both defined over $K$. If $W$ is in normal form, then $W$ has an $(m, D)$-generic in $\mathcal{U}$. 
Proof:

We will construct a differential field containing $K$, and which contains an $(m, D)$ generic of $W$. We work in some large algebraically closed field containing $K$, and choose a generic $\left(a, b_{1}, \cdots, b_{m}\right)$ of $W$ over $K$. Since $W$ is in normal form, by 2.6 we define $D: K\left(a, b_{1}, \cdots, b_{m-1}\right) \rightarrow K\left(a, b_{1}, \cdots, b_{m}\right)$ by setting $D a=b_{1}$ and $D b_{i}=b_{i+1}$, and so that it satisfies $(*)$ and $(* *)$.

Let $v_{m} \subset D^{m} a$ be a transcendence basis of $b_{m}$ over $K\left(a, b_{1}, \cdots, b_{m-1}\right)$ and let $\left(v_{n}\right)_{n>m}$ be a set of tuples of the same length as $v_{m}$ such that for all natural $n>m$ the elements of $v_{n}$ are algebraically independent over $K\left(a, b_{1}, \cdots, b_{m}, v_{m+1}, \cdots, v_{n-1}\right)$. By 2.7 , the map $D$ on $K\left(a, b_{1}, \ldots, b_{m-1}\right)$ extends (uniquely) to a map $D_{1}$ defined on $L=K\left(a, b_{1}, \cdots, b_{m}, v_{n}\right)_{n>m}$ which sends $v_{n}$ to $v_{n+1}$ for $n \geq m$ and satisfies $(*)$ and $(* *)$. Then $D_{1}$ is a derivation of $L$, and $a$ is an $(m, D)$-generic of $W$.

Corollary 2.9 Let $(K, D)$ be a differentially closed field. Let $V$ be a smooth variety, and $W$ a subvariety of $\tau_{m}(V)$ both defined over $K$. Then $W$ is in normal form if and only if $\left\{\left(x, D x, \cdots, D^{m} x\right): x \in V\right\} \cap W$ is Zariski dense in $W$. In particular $\left\{\left(x, D x, \cdots, D^{m} x\right)\right.$ : $x \in V\}$ is Zariski dense in $\tau_{m}(V)$, and $\operatorname{dim}\left(\tau_{m}(V)\right)=(m+1) \operatorname{dim} V$

Remark 2.10 Let $(K, D)$ be a differentially closed field and $V$ a smooth variety in the affine space of dimension $n$ defined over $E=\operatorname{acl}_{D}(E) \subset K$. If $W \subset \tau_{m}(V)$ is a variety in normal form then all $(m, D)$-generics of $W$ have the same type over $E$.

We introduced varieties in normal form to bypass some difficulties concerning differential ideals.

Let $W \subset \tau_{m}(V)$ be a variety in normal form, and let $I \subset K\left[X, Y_{1}, \ldots, Y_{m}\right]$ its defining ideal, which is a prime ideal. Let $\varphi: K\left[X, Y_{1}, \ldots, Y_{m}\right] \rightarrow K[X]_{D}$ be the $K[X]$-algebra embedding sending $Y_{i}$ to $D^{i} X$ for $i=1, \ldots, m$, and let $J$ be the differential ideal generated by $\varphi(I)$.

Let $L$ be a sufficiently saturated differentially closed field containing $K$, and consider the set $\bar{W}$ defined by $J$. The set $\bar{W}$ may not be irreducible for the Kolchin topology. However, it will have an irreducible component $W_{0}$ with the following property: $I_{D}\left(W_{0}\right)$ is the unique prime differential ideal containing $\varphi(I)$ and whose intersection with $K\left[X, D X, \ldots, D^{m} X\right]$ equals $\varphi(I)$. All points in the other irreducible components of $W$ will satisfy some additional equations of order $m$. Furthermore, if $a$ is a generic of $W_{0}$ over $K$ in the sense of the Kolchin topology (i.e., $W_{0}$ is the smallest Kolchin closed set defined over $K$ which contains $a$ ), then $a$ will be an $(m, D)$-generic of $W$ and conversely.

Thus to each variety in normal form defined over $K$ is associated in a canonical way an irreducible Kolchin closed set defined over $K$ (and therefore a unique complete type over $K)$. The condition of a variety being in normal form is clearly expressible by first-order formulas on the coefficients of the defining polynomials, while it is not as immediate that the property of differential polynomials to generate a prime differential ideal is elementary in their coefficients. 
Lemma 2.11 Let $(L, D)$ be a differential field, and let $K$ be a differential subfield of $L$. Let $a$ be a tuple of $L$, let $v \subset a$. If the elements of $D^{m+1} v$ are algebraically independent over $K\left(a, \cdots, D^{m} a\right)$, then for all $i \in\{0, \cdots, m\}$, the elements of $D^{i} v$ are algebraically independent over $K\left(a, \cdots, D^{i-1} a\right)$ (or over $K$ if $i=0$ ).

Proof: By reverse induction on $i$ it is enough to prove that the elements of $D^{m} v$ are algebraically independent over $K\left(a, \cdots, D^{m-1} a\right)$.

If the elements of $D^{m} v$ are algebraically dependent over $K\left(a, \cdots, D^{m-1} a\right)$, then there is a non zero polynomial $P(X) \in K\left(a, \cdots, D^{m-1} a\right)[X]$ which is irreducible and vanishes at $D^{m} v$. Thus $J_{P}\left(D^{m} v\right)\left(D^{m+1} v\right)^{t}+P^{D}\left(D^{m} v\right)=0$, and, as $P$ is irreducible and we work in characteristic zero, $J_{P}\left(D^{m} v\right) \neq 0$. Then, since $P^{D}\left(D^{m} v\right) \in K\left(a, \cdots, D^{m} a\right), D^{m+1} v$ satisfies a non-trivial equation over $K\left(a, \cdots, D^{m} a\right)$ which contradicts our assumption. Hence the elements of $D^{m} v$ are algebraically independent over $K\left(a, \cdots, D^{m-1} a\right)$.

Corollary 2.12 Let $K$ be a differential subfield of $(L, D)$, let a be a tuple of $L$, let $d_{n+1}=$ $\operatorname{tr} . d g\left(D^{n+1} a / K\left(a, \cdots, D^{n} a\right)\right)$. Then $\left(d_{n}\right)_{n \in \mathbb{N}}$ is a decreasing sequence.

Proof:

Let $n \in \mathbb{N}$. Then we have

$$
d_{n+1}=\operatorname{tr} . d g\left(D^{n+1} a / K\left(a, \cdots, D^{n} a\right)\right)=\operatorname{tr} . d g\left(D^{n+1} a / K\left(a, \cdots, D^{n-1} a\right)\left(D^{n} a\right)\right)
$$

and the latter, by 2.11 , is less or equal to $\operatorname{tr} \cdot d g\left(K\left(a, \cdots, D^{n} a\right) / K\left(a, \cdots, D^{n-1} a\right)\right)=d_{n}$.

Remark 2.13 Since $d_{n}$ is a decreasing sequence in $\mathbb{N} \cup\{\infty\}$, there is $M \in \mathbb{N}$ such that $d_{n}=d_{M}$ for all $n \geq M$. Thus $a$ is an $(M, D)$-generic of the locus of $\left(a, D a, \cdots, D^{M} a\right)$ over $K$.

Lemma 2.14 Let $(K, D)$ a differential field and $(L, D)$ an extension. Let $b$ be a tuple of $L$.

Assume that, for $i>1$, tr.dg $\left(D^{i} b / K\left(b, D b, \cdots, D^{i-1} b\right)\right)=\operatorname{tr} . d g(D b / K(b))$. Let $a \in$ $K(b)$ such that, for some $n>\operatorname{tr} . d g(b / K(a))$ we have

tr.dg $\left(\left(D a, \cdots, D^{n} a\right) / K(a)\right)=n t r . d g(D a / K(a))$.

Then tr.dg $\left(\left(D a, \cdots, D^{i} a\right) / K(a)\right)=i \operatorname{tr} . d g(D a / K(a))$ for every $i>n$.

Proof:

We proceed by induction on $d=\operatorname{tr} . d g(D a / K(a))$. It is clear for $d=0$.

Let $v \subset D a$ be a transcendence basis for $D a$ over $K(a)$. We can rewrite the hypothesis of the theorem as: the elements of $\left\{D^{j} v: 0 \leq j<n\right\}$ are algebraically independent over $K(a)$. And we must prove that the elements of $\left\{D^{j} v: j \in \mathbb{N}\right\}$ are algebraically independent over $K(a)$.

Since $\operatorname{tr} . d g\left(v, \cdots, D^{n-1} v / K(a)\right)>\operatorname{tr} . d g(b / K(a)), K\left(a, v, \cdots, D^{n-1} v\right) \not \subset K(b)$. Let $i$ be the smallest integer such that $D^{i} v \not \subset K(b)$; then $D^{i} v \subset K(b, D b)$. Let $w_{0} \subset D^{i} v$ be a 
transcendence basis for $D^{i} v$ over $K(b)$, and let $w \supset w_{0}$ be a transcendence basis for $D b$ over $K(b)$. Let $v_{0} \subset v$ be such that $D^{i} v_{0}=w_{0}$, let $a_{0} \subset a$ be such that $D a_{0}=v_{0}$ and let $v_{1}=v \backslash v_{0}$.

Our hypothesis implies that $\left\{v, \cdots, D^{n-1} v\right\}$ is a transcendence basis for $K\left(a, \cdots, D^{n} a\right)$ over $K(a)$; so $\left\{v_{1}, D v_{1} \cdots, D^{n-1} v_{1}\right\}$ is a transcendence basis for $K\left(a, D a, \cdots, D^{n} a\right)$ over $K\left(a, v_{0}, \cdots, D^{n-1} v_{0}\right)$. Both fields are contained in $K\left(b, w, \cdots, D^{n-i-1} w\right)$, thus the elements of $\left\{D^{j} w: j \geq n-i\right\}$ are algebraically independent over $K\left(a, \cdots, D^{n} a\right)$, so we have $\operatorname{tr} . d g\left(D a, \cdots, D^{n} a /\left(K\left(a_{0}\right)_{D}(a)\right)=\operatorname{tr} . d g\left(D a, \cdots, D^{n} a / K\left(a, D a_{0}, \cdots, D^{n} a_{0}\right)\right)=\right.$ $n t r . d g\left(D a / K\left(a, D a_{0}\right)\right)$.

By induction hypothesis applied to $a$ and $K\left(a_{0}\right)_{D}$, the elements of $\left\{D^{j} v_{1}: j \geq 0\right\}$ are algebraically independent over $K\left(a_{0}\right)_{D}(a)$; thus the elements of $\left\{D^{j} v: j \geq 0\right\}$ are algebraically independent over $K(a)$, since $\operatorname{tr} . d g\left(a_{0}, \cdots, D^{i} a_{0} / K\right)=(i+1) \operatorname{tr} . d g\left(a_{0} / K\right)$ for all $i>0$.

Corollary 2.15 Let $V, W, V_{1} \subset \tau_{m}(V), W_{2} \subset \tau_{m}(W)$ be irreducible varieties defined over a differentially closed field $K$. Let $f: V \rightarrow W$ be a rational map. Then the following property is expressible in the first order language $\mathcal{L}_{D}$ with the parameters needed to define $f, V, W, V_{1}, W_{1}$ :

$V$ and $W$ are smooth varieties, $V_{1}$ and $W_{1}$ are varieties in normal form, and an $(m, D)$-generic of $V_{1}$ is sent by $f$ to an $(m, D)$-generic of $W_{1}$.

Proof:

By the results in [15], we know that we can express in $\mathcal{L}_{D}$ that $V$ is a smooth variety, $V_{1}$ is a variety in normal form, and that a rational map between two varieties sends generic points to generic points.

Using the characterization of varieties in normal form given in 2.3 , for every $m \geq 0$ we can construct subvarieties $V_{m} \subset \tau_{m}(V)$ and $W_{m} \subset \tau_{m}(W)$ such that the $(m, D)$-generics of $V_{m}$ are exactly the $(1, D)$-generics of $V_{1}$ and similarly for $W_{m}$ and $W_{1}$. By 2.14 it suffices to say that the projection $W_{m} \rightarrow V_{m}$ is dominant, where $m=\operatorname{dim}(W)-\operatorname{dim}(V)+1$.

Now we recall some properties of difference fields and its model theory, for a more detailed exposition on this subject see [1] and [3]. As for differential fields, some of the results that we recall here hold for any characteristic.

Let $(K, \sigma)$ be a difference field (i. e. $\sigma$ is an automorphism of $K$ ). The difference polynomial ring over $K$ in $n$ indeterminates is the ring $K[X]_{\sigma}=K\left[X, \sigma(X), \sigma^{2}(X) \cdots\right]$, where $X=\left(X_{1} \cdots, X_{n}\right)$. We extend $\sigma$ to $K[X]_{\sigma}$ in the obvious way. This map is injective but not surjective.

Let $I$ be an ideal of $K[X]_{\sigma}$. We say that $I$ is a reflexive $\sigma$-ideal if for every $f \in K[X]_{\sigma}$, $f \in I$ if and only if $\sigma(f) \in I$. If, in addition, for every $f \in K[X]_{\sigma}, f^{m} \sigma(f)^{n} \in I$ implies $f \in I$, we say that $I$ is a perfect $\sigma$-ideal. A prime ideal which is a perfect $\sigma$-ideal is called a prime $\sigma$-ideal.

If $I$ is a $\sigma$-ideal, then $\sigma$ induces an endomorphism on $K[X]_{\sigma} / I$. 
Proposition $2.16([5]) K\left[X_{1}, \cdots, X_{n}\right]_{\sigma}$ satisfies the ascending chain condition on perfect $\sigma$-ideals.

We define the $\sigma$-topology on $K^{n}$, as the topology whose basic closed sets are $V_{\sigma}(I)=$ $\left\{x \in K^{n}: f(x)=0 \forall f \in I\right\}$ as basic closed sets, where $I \subset K[X]_{\sigma}$ is a $\sigma$-ideal. Note that if $S \subset K^{n}$, then $I_{\sigma}(S)=\left\{f \in k[X]_{\sigma}: f(x)=0 \forall x \in S\right\}$ is a perfect $\sigma$-ideal, thus the $\sigma$-topology is Noetherian.

Theorem 2.17 The theory of difference fields has a model-companion, that we shall denote by ACFA. It is described as follows.

$(K, \sigma)$ is a model of ACFA if and only if:

1. $K$ is an algebraically closed field.

2. $(K, \sigma)$ is a difference field.

3. For every irreducible algebraic variety $V$, if $W$ is an irreducible algebraic subvariety of $V \times V^{\sigma}$, such that the projections from $W$ onto $V$ and $V^{\sigma}$ are dominant, then there is $a \in V(K)$ such that $(a, \sigma(a)) \in W$.

Here $V^{\sigma}$ denotes the variety obtained by applying $\sigma$ to the polynomials defining $V$.

Let $(K, \sigma)$ a difference field, $A \subset K$. We denote by $\operatorname{cl}_{\sigma}(A)$ the smallest difference field containing $(A)$, and by $a c l_{\sigma}(A)$ the field-theoretic algebraic closure of $c l_{\sigma}(A)$.

If $(K, \sigma)$ be a model of $A C F A$, then $\operatorname{acl}_{A C F A}(A)=\operatorname{acl}_{\sigma}(A)$.

$A C F A$ is model-complete, it is not complete but its completions can easily be described and all of them are supersimple and eliminates imaginaries.

\section{Difference-Differential Fields}

In this section we introduce the definitions, basic facts and main model-theoretic properties of difference-differential fields of characteristic 0 . We will work in the language $\mathcal{L}_{\sigma, D}=$ $\{0,1,+,-, \cdot, \sigma, D\}$.

Definition 3.1 A difference-differential ring is a differential ring $(R, D)$ with an automorphism $\sigma$ of the $\mathcal{L}_{D}$-structure $(R, D)$, (i.e. $\sigma$ commutes with $\left.D\right)$.

If $R$ is a field we say that $(R, \sigma, D)$ is a difference-differential field.

Definition 3.2 Let $(R, \sigma, D)$ be a difference-differential ring. The ring of differencedifferential polynomials in $n$ indeterminates over $R$ is the ring $R\left[X_{1}, \cdots, X_{n}\right]_{\sigma, D}$ of polynomials in the variables $\sigma^{i}\left(D^{j} X_{1}\right), \cdots, \sigma^{i}\left(D^{j} X_{n}\right)$ for $i, j \in \mathbb{N}$.

Remark 3.3 As in sections 1 and 2 , we can extend $D$ to a derivation on $R\left[X_{1}, \cdots, X_{n}\right]_{\sigma, D}$ and $\sigma$ to an endomorphism of $R\left[X_{1}, \cdots, X_{n}\right]_{\sigma, D}$ which commutes with $D$.

Definition 3.4 Let $(R, \sigma, D)$ be a difference-differential ring. Let I be an ideal of $R$. 
1. We say that $I$ is a $(\sigma, D)$-ideal if it is a differential ideal and a reflexive $\sigma$-ideal.

2. We say that $I$ is a perfect $(\sigma, D)$-ideal if it is a $(\sigma, D)$-ideal which is perfect as a $\sigma$-ideal.

Notation 3.5 Let $(K, \sigma, D)$ a difference-differential field, $S \subset K[X]_{\sigma, D}$, with $X=\left(X_{1}, \cdots, X_{n}\right)$. Let $A \subset K^{n}, a \in K^{n}$ and let $E$ be a difference-differential subfield of $K$.

1. $V_{\sigma, D}(S)=\left\{x \in K^{n}: \forall f(X) \in S f(x)=0\right\}$.

2. $I_{\sigma, D}(A)=\left\{f(X) \in K[X]_{\sigma, D}: \forall x \in A f(x)=0\right\}$.

3. $I_{\sigma, D}(a / E)=\left\{f(X) \in E[X]_{\sigma, D}: f(a)=0\right\}$.

We define the $(\sigma, D)$-topology on $K^{n}$ to be the topology with the sets of the form $V_{\sigma, D}(S)$ as a basis of closed sets.

Remark 3.6 Let $(K, \sigma, D)$ be a difference-differential field, $A \subset K^{n}$. Then $I_{\sigma, D}(A)$ is a perfect $(\sigma, D)$-ideal.

Now we need some extra definitions. For the details see [4].

Definition 3.7 Let $R$ be a commutative ring, and let $\mathcal{C}$ a set of ideals of $R$.

1. We say that $\mathcal{C}$ is a conservative system of ideals if:

(a) For every $A \subset \mathcal{C}, \cap A \in \mathcal{C}$.

(b) For every $A \subset \mathcal{C}$ totally ordered by inclusion, $\cup A \in \mathcal{C}$.

2. Let $\mathcal{C}$ be a conservative system of ideals. We say that $C$ is divisible if for $I \in \mathcal{C}$ and $a \in R$ we have $(I: a) \in \mathcal{C}$.

3. Let $\mathcal{C}$ be a divisible conservative system of ideals. We say that $\mathcal{C}$ is perfect if all its members are radical ideals.

The following is proved in [4] (pp. 798-799).

Proposition 3.8 Let $(R, \sigma, D)$ be a difference-differential ring. The set of perfect $(\sigma, D)$ ideals of $R$ is perfect in the sense of 3.7 and it contains any perfect set of ideals.

Theorem 3.9 ([4], section 2, Theorem I) Let $R$ be a commutative ring and $\mathcal{C}$ a perfect system of ideals of $R$, let $I \in \mathcal{C}$. Then $I$ is an intersection of prime ideals of $\mathcal{C}$. If $\mathcal{C}$ is Noetherian this intersection can be taken finite.

Theorem 3.10 ([5], section 5, Corollary I) Let $(R, \sigma, D)$ a difference-differential ring which contains $\mathbb{Q}$ and such that the set of perfect $(\sigma, D)$-ideals of $R$ satisfies the ascending chain condition. Let $(S, \sigma, D)$ be a difference-differential ring finitely generated over $R$ as a difference-differential ring. Then the set of perfect $(\sigma, D)$-ideals of $S$ satisfies the ascending chain condition. 
Corollary 3.11 Let $(K, \sigma, D)$ be a difference-differential field. Then the $(\sigma, D)$-topology of $K^{n}$ is Noetherian.

Corollary 3.12 Let $(K, \sigma, D)$ be a difference-differential field and let I be a perfect $(\sigma, D)$ ideal of $K\left[X_{1}, \cdots, X_{n}\right]_{\sigma, D}$. Then $I$, as a perfect $(\sigma, D)$-ideal, is generated by finitely many difference-differential polynomials.

Corollary 3.13 Let $(K, \sigma, D)$ be a difference-differential field and let I be a perfect $(\sigma, D)$ ideal of $K\left[X_{1}, \cdots, X_{n}\right]_{\sigma, D}$. Then $I$ is a finite intersection of prime perfect $(\sigma, D)$-ideals.

Now we state Hrushovski theorem, which he proved but never published.

Theorem 3.14 (Hrushovski)

The model companion of the theory of difference-differential fields exists. We denote it DCFA.

Now we give an axiomatization to $D C F A$.

Theorem $3.15(K, D, \sigma)$ is a model of DCFA if

1. $(K, D)$ is a differentially closed field.

2. $\sigma$ is an automorphism of $(K, D)$.

3. If $U, V, W$ are varieties such that:

(a) $U \subset V \times V^{\sigma}$ projects generically onto $V$ and $V^{\sigma}$.

(b) $W \subset \tau_{1}(U)$ projects generically onto $U$.

(c) $\pi_{1}(W)^{\sigma}=\pi_{2}(W)$ (we identify $\tau_{1}\left(V \times V^{\sigma}\right)$ with $\left.\tau_{1}(V) \times \tau_{1}(V)^{\sigma}\right)$ and let $\pi_{1}$ : $\tau_{1}\left(V \times V^{\sigma}\right) \rightarrow \tau_{1}(V)$ and $\pi_{2}: \tau_{1}\left(V \times V^{\sigma}\right) \rightarrow \tau_{1}(V)^{\sigma}$ be the natural projections $)$.

(d) $A(1, D)$-generic point of $W$ projects onto a $(1, D)$-generic point of $\pi_{1}(W)$ and onto a (1,D)-generic point of $\pi_{2}(W)$.

Then there is a tuple $a \in V(K)$, such that $(a, \sigma(a)) \in U$ and $(a, D a, \sigma(a), \sigma(D a)) \in W$.

Proof:

By 2.15 , these are first order properties.

First we prove that any difference-differential field embeds in a model of $D C F A$. By quantifier elimination of $D C F$ any difference-differential field embeds in a model of (1) and (2). By the usual model-theoretic argument, it suffices to show that any instance of (3) over a difference-differential field $(K, \sigma, D)$ can be realized in an extension of $(K, \sigma, D)$.

Let $(K, \sigma, D)$ be a difference-differential field such that $K=A C F$. Let $U, V, W$ be $K$ varieties satisfying $(3)$. Let $(\mathcal{U}, D)$ be a saturated model of $D C F$ containing $(K, D)$. Let $(a, b)$ be a $(1, D)$-generic of $W$; then $a$ is a $(1, D)$-generic of $\pi_{1}(W)$ and $b$ is a $(1, D)$-generic 
of $\pi_{1}(W)^{\sigma}$. Hence $t p_{D C F}(b / K)=\sigma\left(t p_{D C F}(a / K)\right)$; thus $\sigma$ extends to an automorphism $\sigma^{\prime}$ of $(\mathcal{U}, D)$ such that $\sigma^{\prime}(a)=b$.

Now we shall prove that the models of $D C F A$ are existentially closed. Let $(K, \sigma, D)$ be a model of $D C F A$ contained in a difference-differential field $(\mathcal{U}, \sigma, D)$. Since $x \neq 0 \leftrightarrow$ $\exists y x y=1$, it suffices to prove that every finite system of $(\sigma, D)$-polynomial equations with coefficients in $K$ with a solution in $\mathcal{U}$ has a solution in $K$. Let $\varphi(x)$ be such a system and let $a$ be a tuple of $\mathcal{U}$ satisfying $\varphi$. Since $\sigma$ is an automorphism, $\varphi$ is a finite conjunction of equations of the form $f\left(x, \cdots, \sigma^{n}(x)\right)=0$, where $f$ is a differential polynomial; such an equation is equivalent, modulo the theory of difference-differential fields, to a formula of the form:

$$
\exists y_{0}, \cdots, y_{k-1} f\left(y_{0}, \cdots, y_{k-1}, \sigma\left(y_{k-1}\right)\right)=0 \wedge \bigwedge_{i=1}^{k-1}\left(y_{i}=\sigma\left(y_{i-1}\right) \wedge y_{0}=x\right) .
$$

Thus, if we replace $x$ by $\left(y_{0}, \cdots, y_{k-1}\right)$ and $a$ by $\left(a, \cdots, \sigma^{k-1}(a)\right)$, we may suppose that $\varphi$ is a finite conjunction of equations of the form $g(x, \sigma(x))=0$, where $g(X, Y)$ is a differential polynomial over $K$.

Let $m$ be sufficiently large so that $X$ and $Y$ appear in each $g(X, Y)$ with differential order less than $m$, and such that, for $M>m$

$$
\begin{aligned}
& \operatorname{tr} . d g\left(\left(D^{M+1} a, D^{M+1} \sigma(a)\right) / K\left(a, \sigma(a), \cdots, D^{M} a, D^{M} \sigma(a)\right)\right)= \\
& \quad \operatorname{tr} . d g\left(\left(D^{m} a, D^{m} \sigma(a)\right) / K\left(a, \sigma(a), \cdots, D^{m-1} a, D^{m-1} \sigma(a)\right)\right)
\end{aligned}
$$

and

$$
\operatorname{tr} . d g\left(D^{M+1} a / K\left(a, \cdots, D^{M} a\right)\right)=\operatorname{tr} . d g\left(D^{m} a / K\left(a, \cdots, D^{m-1} a\right)\right) .
$$

Let $V$ be the locus of $b=\left(a, D a, \cdots, D^{m} a\right)$ over $K, U$ the locus of $(b, \sigma(b))$ over $K$, and let $W \subset \tau_{1}\left(V \times V^{\sigma}\right)$ be the locus of $(b, D b, \sigma(b), \sigma(D b))$ over $K$. By construction and choice of $m, b$ is a $(1, D)$-generic of $\pi_{1}(W), \sigma(b)$ is a $(1, D)$-generic of $\pi_{2}(W)$ and $(b, \sigma(b))$ is a $(1, D)$-generic of $W$. By axiom (3) there is a tuple $c=\left(c_{0}, \cdots, c_{m}\right)$ in $K$ such that $(c, D c, \sigma(c), \sigma(D c)) \in W$. Thus $\left(c_{0}, \sigma\left(c_{0}\right)\right)$ satisfies all the equations of differential order less than or equal to $m$ satisfied by $(a, \sigma(a))$; hence $c_{0}$ satisfies $\varphi(x)$.

Example 3.16 The following shows why we need the $(1, D)$-generics in our axioms, generics are not strong enough to describe differential types. Consider the set $A$ defined by the equations $\sigma(x)=D x$ and $D \sigma(x)=x^{2}$. It is then given by a subvariety $W \subset \tau\left(\mathbb{A}^{1}\right) \times \tau\left(\mathbb{A}^{1}\right)$ which is defined by the equations $x_{2}=y_{1}$ and $x_{1}^{2}=y_{2}$. The variety $W$ projects on each copy of $\tau\left(\mathbb{A}^{1}\right)$.

Let $a \in A, a \neq 0$. From $\sigma(a)=D a$ one deduces that $\sigma^{i} D^{j} a=\sigma^{i+j} a=D^{i+j} a$ for all $i, j \in \mathbb{N}$. Thus $\sigma^{3}(a)=(D a)^{2}=2 a D a$, which implies that $D a=2 a$. Thus there are differential relations that cannot be seen from the defining equations.

Remark 3.17 If $(K, D, \sigma)$ is a model of DCFA then $(K, \sigma)$ is a model of ACFA. 
Proof:

Take $W=\tau_{1}(U)$, and apply 3.15 .

Proposition 3.18 Let $(K, \sigma, D)$ be a model of DCFA, and I a perfect $(\sigma, D)$-ideal. Then $I_{\sigma, D}(V(I))=I$

Proof:

Clearly $I \subset I_{\sigma, D}(V(I))$. Let $f \in K[X]_{\sigma, D}$, such that $f \notin I$. By 3.13, there is a prime perfect $(\sigma, D)$-ideal $J$ containing $I$ such that $f \notin J$. Then $K[\bar{X}]_{\sigma, D} / J$ embeds in difference-differential field $L$.

By 3.12, $J$ is generated by a finite tuple of polynomials $P(\bar{X})$. Let $\bar{a}$ be the image of $\bar{X}$ in $L$. Thus we have that $L \models P(\bar{a})=0$ and $L \models f(\bar{a}) \neq 0$. Since $(K, \sigma, D)$ is existentially closed there is $\bar{b} \in K$ such that $P(\bar{b})=0$ and $f(\bar{b}) \neq 0$. But $I \subset J$, thus $\bar{b} \in V(I)$, which implies $f \notin I_{\sigma, D}(V(I)$

Definition 3.19 Let $E \subseteq F$ be two difference-differential fields, let $a \in F$.

1. We define $\operatorname{deg}_{\sigma, D}(a / E)$ to be the transcendence degree of $E(a)_{\sigma, D}$ over $E$ if it is finite, in this case we say that a is finite-dimensional over $E$, otherwise we set $\operatorname{deg}_{\sigma, D}(a / E)=\infty$ and we say that a is infinite-dimensional over $E$.

2. If $I_{\sigma, D}(a / E)=(0)$ we say that a is $(\sigma, D)$-transcendental over $E$, otherwise we say that it is $(\sigma, D)$-algebraic over $E$.

Remark 3.20 If a is $(\sigma, D)$-algebraic over $E$ it is not always true that $\operatorname{deg}_{\sigma, D}(a / E)$ is finite.

Remark 3.21 There is a natural notion of $(\sigma, D)$-transcendence basis.

For the following results see [2], sections 2 and 3 .

Proposition 3.22 Let $K_{1}, K_{2}$ be models of DCFA, let $E$ an algebraically closed differencedifferential subfield of $K_{1}$ and $K_{2}$. Then $K_{1} \equiv_{E} K_{2}$.

Corollary 3.23 Let $E$ be an algebraically closed difference-differential field, then DCFA $\cup q f \operatorname{Diag}(E)$ is complete.

Corollary 3.24 Let $\left(K_{1}, \sigma_{1}, D_{1}\right),\left(K_{2}, \sigma_{2}, D_{2}\right)$ be two models of DCFA containing a common difference-differential field $(E, \sigma, D)$. Then $K_{1} \equiv_{E} K_{2}$ if and only if $\left(E^{\text {alg }}, \sigma_{1}, D_{1}\right) \simeq$ $\left(E^{a l g}, \sigma_{2}, D_{2}\right)$.

Let $(K, \sigma, D)$ be a differential-difference field, $A \subset K$. We denote by $c l_{\sigma, D}(A)$ the smallest difference-differential field containing $A$, and by $\operatorname{acl}_{\sigma, D}(A)$, the field-theoretic algebraic closure of $c l_{\sigma, D}(A)$. 
Corollary 3.25 Let $E$ be a difference-differential subfield of a model $K$ of DCFA . Let $a, b$ be tuples of $K$. Then $t p(a / E)=t p(b / E)$ if and only if there is an E-isomorphism between $\operatorname{acl}_{\sigma, D}(E(a))$ and $\operatorname{acl}_{\sigma, D}(E(b))$ which sends a to $b$.

Corollary 3.26 Let $\phi(\bar{x})$ be a formula. Then, modulo DCFA, $\phi(\bar{x})$ is equivalent to a disjunction of formulas of the form $\exists \bar{y} \psi(\bar{x}, \bar{y})$, where $\psi$ is quantifier free, and for every tuple $(\bar{a}, \bar{b})$ in a difference-differential field $K$ satisfying $\psi, \bar{b} \in \operatorname{acl}_{\sigma, D}(\bar{a})$.

Proposition 3.27 Let $(K, \sigma, D)$ be a model of DCFA. Let $A \subset K$. Then the (modeltheoretic) algebraic closure $\operatorname{acl}(A)$ of $A$ is $\operatorname{acl}_{\sigma, D}(A)$.

Proposition 3.27 allows us to define the independence relation in difference-differential fields in terms of independence relation for fields.

Definition 3.28 Let $K$ be a model of DCFA, let $A, B, C$ be subsets of $K$. We say that $A$ is independent from $B$ over $C$, denoted $A \downarrow_{C} B$, if acl $(A, C)$ is linearly disjoint from $\operatorname{acl}(B, C)$ over $\operatorname{acl}(C)$.

Remark 3.29 The independence relation, in a model $(K, \sigma, D)$ of $D C F A$, has the following properties.

1. Let a be a tuple of $K$, and $A, B \subset K$ such that $a \downarrow_{C} B$; let $\tau$ be an automorphism of $(K, \sigma, D)$. Then $\tau(a) \downarrow_{\tau(C)} \tau(B)$.

2. For all finite tuple $a$, and for all $B$ there is a finite subset $C$ of acl $(B)$ such that $a \downarrow_{C} B$

3. For all tuple $a$, for all set $B$ and for all $C$ containing $B$ there is a tuple $a^{\prime}$ such that $t p(a / B)=t p\left(a^{\prime} / B\right)$ and $a \downarrow_{B} C$.

4. For all tuples $a, b$ and for all $C, a \downarrow_{C} b$ if and only if $b \downarrow_{C} a$.

5. Let $a$ be a tuple and let $A \subset B \subset C$. Then $a \downarrow_{B} C$ and $a \downarrow_{A} B$ if and only if $a \downarrow_{A} C$.

The following are direct consequences of the results in [6].

Theorem 3.30 1. The independence relation defined above coincides with nonforking.

2. Every completion of DCFA is supersimple.

This theorem implies in particular that $D C F A$ satisfies the Independence Theorem over models; however, as in $A C F A$, we will show that $D C F A$ satisfies the Independence Theorem over algebraically closed structures, with a little more work one could also show that $D C F A$ satisfies the Generalized Independence Theorem over algebraically closed structures.

Theorem 3.31 If $\mathcal{U}$ is a saturated model of DCFA, $E$ an algebraically closed subset of $\mathcal{U}$, and $\bar{a}, \bar{b}, \bar{c}_{1}, \bar{c}_{2}$ tuples in $\mathcal{U}$ such that: 
1. $\operatorname{tp}\left(\bar{c}_{1} / E\right)=\operatorname{tp}\left(\bar{c}_{2} / E\right)$.

2. $\bar{a} \downarrow_{E} \bar{c}_{1}, \bar{a} \downarrow_{E} \bar{b}$ and $\bar{b} \downarrow_{E} \bar{c}_{2}$.

Then there is $\bar{c}$ realizing $t p\left(\bar{c}_{1} / E \cup \bar{a}\right) \cup t p\left(\bar{c}_{2} / E \cup \bar{b}\right)$ such that $\bar{c} \downarrow_{E}(\bar{a}, \bar{b})$.

Proof:

Let $\bar{c}$ be a realization of $t p\left(\bar{c}_{1} / E\right)$ such that $\bar{c} \downarrow_{E}(\bar{a}, \bar{b})$. Let $A=\operatorname{acl}(E \bar{a}), B=$ $\operatorname{acl}(E \bar{b}), C=\operatorname{acl}(E \bar{c})$. Let $\phi_{1}: \operatorname{acl}\left(E \bar{c}_{1}\right) \rightarrow C$ and $\phi_{2}: \operatorname{acl}\left(E \bar{c}_{2}\right) \rightarrow C$ two $\mathcal{L}_{\sigma, D}(E)-$ isomorphisms such that $\phi_{i}\left(\bar{c}_{i}\right)=\bar{c}$.

Let $\sigma_{0}=\left.\sigma\right|_{(A B)^{a l g} C}$. Since $A$ is linearly disjoint from $\operatorname{acl}\left(E \bar{c}_{1}\right)$ and from $C$ over $E$, we can extend $\phi_{1}$ to a $\mathcal{L}_{D}(A)$-isomorphism $\psi_{1}$ between $\operatorname{acl}\left(A \bar{c}_{1}\right)$ and $(A C)^{\text {alg }}\left(=\operatorname{acl}_{D}(A C)\right)$. Let $\sigma_{1}=\psi_{1} \sigma \psi_{1}^{-1} ; \sigma_{1}$ is an automorphism of $(A C)^{\text {alg }}$ and agrees with $\sigma$ on $A$ and $C$. By definition of $\sigma_{1}, \psi_{1}$ is a $\mathcal{L}_{\sigma, D}(A)$-isomorphism between $\left(\operatorname{acl}\left(A \bar{c}_{1}\right), \sigma\right)$ and $\left((A C)^{a l g}, \sigma_{1}\right)$. In the same way we define $\psi_{2}: \operatorname{acl}\left(B c_{2}\right) \rightarrow(B C)^{\text {alg }}$ and $\sigma_{2} \in A u t(B C)^{\text {alg }}$.

Let $L=(A B)^{\text {alg }}(A C)^{\text {alg }}(B C)^{\text {alg }}$ (which is a differential field that extends $\left.A, B, C\right)$. Let us suppose that there is an $\mathcal{L}_{D}$-automorphism $\tau$ of $L$ which extends $\sigma_{0}, \sigma_{1}, \sigma_{2}$. Let $\left(M, \tau^{\prime}, D\right) \models D C F A$ contain $(L, \tau, D)$. Since $\tau$ extends $\sigma_{0}$, by 3.22 , we have $t p_{M}(A B / E)=$ $\operatorname{tp}_{\mathcal{U}}(A B / E)$; since $\tau$ extend $\sigma_{i}$, the $\psi_{i}$ 's are difference-differential field isomorphisms. Applying 3.25 we have $t p_{M}(\bar{c} / A)=t p_{\mathcal{U}}\left(\bar{c}_{1} / A\right)$ and $t p_{M}(\bar{c} / B)=t p_{\mathcal{U}}\left(\bar{c}_{1} / B\right)$. Also $\bar{c} \downarrow_{E}(A, B)$. Hence to finish the proof, all we have to do is show the existence of such a $\tau$. To do this, we will prove that $\sigma_{0}, \sigma_{1}$ have a unique extension $\tau_{1}$ to $(A B)^{\text {alg }}(A C)^{\text {alg }}$, and that there is an extension $\tau_{2}$ of $\tau_{1}, \sigma_{2}$ to $L$ (Note that these automorphisms will commute with $D$ ).

For the first part it is enough to show that $(A B)^{a l g} C$ is linearly disjoint from $(A C)^{a l g}$ over $(A B)^{\text {alg }} C \cap(A C)^{\text {alg }}$, and that $\sigma_{0}$ and $\sigma_{1}$ agree on $(A B)^{\text {alg }} C \cap(A C)^{\text {alg }}$. Similarly for the second part.

By Remark 2 of 1.9 in [1], we have

$$
(A B)^{a l g} C \cap(A C)^{a l g}=A C(*), \quad(A B)^{a l g}(A C)^{a l g} \cap(B C)^{a l g}=B C(* *) .
$$

Since $(A C)^{a l g}$ is Galois over $A C$ it implies that $(A C)^{a l g}$ and $(A B)^{a l g}$ are linearly disjoint over $A C$; as $\sigma_{0}$ and $\sigma_{1}$ both extend $\sigma$ on $A C$, they are compatible. The same argument applies for the second part.

A first order theory $T$ (in a language $\mathcal{L}$ ) is called quantifier-free $\omega$-stable if for any saturated model $M$ of $T$, there are only countably many quantifier free types over a countable set. Let $T$ be such a theory which eliminates quantifiers and imaginaries, let $\mathcal{L}_{\sigma}=\mathcal{L} \cup\{\sigma\}$ where $\sigma$ is an 1-ary fucntion symbol. Let $T_{0}$ be the $\mathcal{L}_{\sigma}$-theory whose models are the structures of the form $(M, \sigma)$ where $M$ is a model of $T$ and $\sigma$ is an automorphism of $M$. Assume that $T$ has a model-companion $T_{A}$. Let $(M, \sigma)$ be a model of $T_{0}$. Let $A=\operatorname{acl}(A) \subset M$ and let $a \in M$. Then $q \operatorname{ftp}_{\mathcal{L}_{\sigma}}(a / A)$ is entirely determined by $t p_{T}\left(\left(\sigma^{i}(a)\right)_{i \in \mathbb{Z}} / A\right)$. Let $B=d c l_{T}\left(A, \sigma^{-i}(a) \mid i>0\right)$, and consider $t p_{T}(a / B)$. As $T$ is $\omega$-stable, there is some integer $n$ such that $t p_{T}(a / B)$ is the unique non-forking extension of $t p_{T}\left(a / A, \sigma^{-1}(a), \ldots, \sigma^{-n}(a)\right)$ to $B$. Applying $\sigma^{i}$, this gives that $t p_{T}\left(\sigma^{i}(a) / \sigma^{i}(B)\right)$ is the 
unique non-forking extension of $t p_{T}\left(\sigma^{i}(a) / A, \sigma^{i-1}(a), \ldots, \sigma^{i-n}(a)\right)$ to $\sigma^{i}(B)$. This implies that $T_{0}$ is quantifier-free- $\omega$-stable (and so is $T_{A}$ ).

Remark 3.32 As DCF is $\omega$-stable, DCFA is quantifier-free $\omega$-stable.

Now we want to prove that $D C F A$ eliminates imaginaries. We shall need some properties of the fundamental order for types in stable theories. The definitions and proofs can be found in [14].

Recall that a type $p(x)$ over some set $A$ represents the $\mathcal{L}$-formula $\phi(x, y)$ if there is a tuple $a \in A$ such that $\phi(x, a) \in p(x)$. We denote by $\beta(p)$ the set of formulas represented by $p$.

For convenience, we will define the fundamental order on types whose domain is algebraically closed, so that they are stationary (and definable by elimination of imaginaries in $D C F$ ).

Definition 3.33 Let $A$ and $B$ be algebraically closed differential subfields of some model $(\mathcal{U}, D)$ of $D C F$, and let $p(x), q(x)$ be types over $A$ and $B$ respectively. We write $p \leq_{\text {fo }} q$ if $\beta(q) \subseteq \beta(p)$, and $\beta(p) \sim_{f o}$ if $\beta(p)=\beta(q) . \leq_{\text {fo }}$ is called the fundamental order.

Proposition 3.34 If $A \subset B$ and $q$ is an extension of $p$, one has $q \leq_{\text {fo }} p$, and $q \sim_{f o} p$ if and only if $q$ is a non-forking extension of $p$.

If $p$ and $q$ are types in an infinite number of variables $\left(x_{i}\right)_{i \in I}$ we say that $p \leq_{f o} q$ if and only if for every finite $J \subset I$, if $p^{\prime}$ and $q^{\prime}$ denote the restrictions of $p$ and $q$ to the variables $\left(x_{i}\right)_{i \in J}$, we have $p^{\prime} \leq_{f o} q^{\prime}$.

Remark $3.35 \sim_{f o}$ is an equivalence relation on the class of types in the variables $\left(x_{i}\right)_{i \in I}$.

Proposition 3.36 Every completion of DCFA eliminates imaginaries.

Proof:

Let $(K, \sigma, D)$ be a saturated model of $D C F A$, let $\alpha \in K^{e q}$. Then there is a $\emptyset$-definable function $f$ and a tuple $a$ in $K$ such that $f(a)=\alpha$.

Let $E=a c l^{e q}(\alpha) \cap K$. If $\alpha$ is definable over $E$, let $b$ be a tuple of $E$ over which $\alpha$ is definable; then $b \in \operatorname{acl}^{e q}(\alpha)$. Since we are working in a field, there is a tuple $c$ of $K$ which codes the (finite) set of conjugates of $b$ over $\alpha$. Hence $c$ and $\alpha$ are interdefinable.

Let us suppose that $\alpha$ is not definable over $E$, in particular, $a$ is not a tuple of $E$. We will show that there is a realization $b$ of $t p(a / \alpha)$ such that $b \downarrow_{E} a$.

We now work in the theory $D C F$, and replace the tuple $a$ by the infinite tuple $\left(\sigma^{i}(a)\right)_{i \in \mathbb{Z}}$, which we also denote by $a$.

Since $\operatorname{tp}(a / \alpha)$ is non-algebraic, it has a realization $b$ such that $\operatorname{acl}^{e q}(a) \cap \operatorname{acl}^{e q}(b)=$ $\operatorname{acl}^{e q}(\alpha)$, and thus

$$
\operatorname{acl}(E a) \cap \operatorname{acl}(E b)=E
$$

Choose such a $b$ such that, if $b^{\prime}$ satisfies the same properties, then $t p_{D C F}\left(b^{\prime} / a c l(E a)\right) \ngtr_{f o}$ $t p_{D C F}(b / a c l(E a))$. 
Let $c$ be a tuple of $K$ such that $\operatorname{tp}(c / a c l(E a))=t p(b / \operatorname{acl}(E a))$, and $c \downarrow_{E a} b$. Then $f(c)=f(a)$ and $c$ satisfies

$$
\operatorname{acl}(E c) \cap \operatorname{acl}(E a b) \subset \operatorname{acl}(E c) \cap \operatorname{acl}(E a)=E,
$$

and there is no $c^{\prime}$ satisfying $(* *)$ such that $t p_{D C F}\left(c^{\prime} / \operatorname{acl}(\operatorname{Eab})\right)>_{f_{o}} t p_{D C F}(c / a c l(E a b))$.

Then $t p(c / a c l(E b)) \geq_{f o} t p(c / a c l(E a b)) \sim_{f o} t p(c / a c l(E a))$. Let $\tau$ be an $\mathcal{L}_{D}(E)$ automorphism sending $b$ to $a$. Then $t p(\tau(c) / a c l(E a)) \sim_{f o} t p(c / a c l(E b))$, and $\tau(c)$ satisfies $(*)$ (by $(* *))$. Hence, by maximality of $t p(b / a c l(E a))=t p(c / a c l(E a))$, we get that $t p(c / a c l(E b)) \sim_{f o} t p(c / a c l(E a))$, and therefore $c \downarrow_{E b} a$. By elimination of imaginaries and $(*)$, this implies that $c \downarrow_{E} a b$, and therefore $a \downarrow_{E} b$.

We have shown that there is a tuple $b$ realizing $t p(a / \alpha)$ independent from $a$ over $E$. But $\alpha$ is not $E$-definable, thus there is $a^{\prime}$ realizing $t p(a / E)$ such that $f(a) \neq f\left(a^{\prime}\right)$, and we may choose it independent from $b$ over $E$. Since $t p(a / E)=t p\left(a^{\prime} / E\right)$, there is a realization $c^{\prime}$ of $t p\left(a^{\prime} / E\right)$ such that $f\left(a^{\prime}\right)=f\left(c^{\prime}\right)$ and $c^{\prime} \downarrow_{E} a^{\prime}$; we may suppose that $c^{\prime} \downarrow_{E} b$. If we apply the independence theorem to $t p(a / E b) \cup t p\left(a^{\prime} / E c^{\prime}\right)$ we get a contradiction.

Lemma 3.37 Let $(K, \sigma, D)$ be a model of DCFA, let $E=\operatorname{acl}(E) \subset K$, and let $(L, \tau, D)$ be a difference-differential field extending $\left(K, \sigma^{n}, D\right)$, where $n$ is a positive integer. Then there is a difference-differential field $\left(M, \sigma^{\prime}, D\right)$ containing $(E, \sigma, D)$ such that $\left(\sigma^{\prime}\right)^{n}$ extends $\tau$.

Proof:

For $i=1, \cdots, n-1$ let $L_{i}$ be a difference-differential field realizing $\sigma^{i}\left(t p_{D C F}(L / E)\right)$ such that $L_{0}=L, L_{1}, \cdots, L_{n-1}$ are linearly disjoint over $E$. Let $f_{0}=i d_{L}$ and for $i=1, \cdots, n-1$ let $f_{i}: L \longrightarrow L_{i}$ be an $\mathcal{L}_{D^{-}}$-isomorphism extending $\sigma^{i}$ on $E$.

For $i=1, \cdots, n-1$ let $\sigma_{i}: L_{i-1} \longrightarrow L_{i}$ be defined by $\sigma_{i}=f_{i} f_{i-1}^{-1}$, and let $\sigma_{n}: L_{n-1} \longrightarrow$ $L_{0}$ be defined by $\sigma_{n}=\tau f_{n-1}^{-1}$.

Let $x \in E$. If $i=1, \cdots, n-1$ then $\sigma_{i}(x)=f_{i}\left(f_{i-1}^{-1}(x)\right)=\sigma^{i}\left(\sigma^{-(i-1)}(x)\right)=\sigma(x)$; and $\sigma_{n}(x)=\tau\left(\sigma^{-(n-1)}(x)\right)=\sigma^{n}\left(\sigma^{-(n-1)}(x)\right)=\sigma(x)$. Hence each $\sigma_{i}$ extends $\sigma$ on $E$.

Also, we have $\sigma_{n} \sigma_{n-1} \cdots \sigma_{1}=\tau\left(f_{n-1}^{-1} f_{n-2}\right) \cdots\left(f_{1} f_{0}^{-1}\right)=\tau f_{0}^{-1}=\tau$.

Let $M$ be the composite of $L_{0}, \cdots, L_{n-1}$. Since the $L_{i}$ 's are linearly disjoint over $E$, $M$ is isomorphic to the quotient field of $L_{0} \otimes_{E} \cdots \otimes_{E} L_{n-1}$. There is a unique derivation on $M$ extending the derivations of the $L_{i}$ 's and there is a unique $\mathcal{L}_{D^{-}}$-automorphism $\sigma^{\prime}$ of $M$ which coincides with $\sigma_{i}$ on $L_{i-1}$.

By the above $\left(\sigma^{\prime}\right)^{n}$ extends $\tau$.

Corollary 3.38 Let $(K, \sigma, D)$ be a model of DCFA. Then, for all $n \in \mathbb{N}\left(K, \sigma^{n}, D\right)$ is a model of DCFA.

Proof:

Let $\Sigma$ be a finite system of $\left(\sigma^{n}, D\right)$-equations over $K$, and let $(L, \tau, D)$ be an extension of $\left(K, \sigma^{n}, D\right)$ containing a solution of $\Sigma$. By 3.37 there is an extension $\left(M, \sigma^{\prime}, D\right)$ of $(K, \sigma, D)$ such that $\left(M,\left(\sigma^{\prime}\right)^{n}, D\right)$ is an extension of $(L, \tau, D)$. Thus, $M$ contains a solution of $\Sigma$, and since $(K, \sigma, D)$ is existentially closed, it conatains a solution of $\Sigma$. 
DIFFERENTIALLY CLOSED FIELDS OF CHARACTERISTIC ZERO

\section{The Field of Constants and the Fixed Field}

In this section we study two special subfields of a model $(K, \sigma, D)$ of $D C F A$ : the differential field $($ Fix $\sigma, D)$ and the difference field $(\mathcal{C}, \sigma)$ where $F i x \sigma$ is the fixed field of $K$ and $\mathcal{C}$ is the field of constants of $K$.

Throughout this section $(K, \sigma, D)$ will denote a model of $D C F A$.

Proposition $4.1(\mathcal{C}, \sigma)$ is a model of $A C F A$.

Proof :

Since $\sigma$ commutes with $D,(\mathcal{C}, \sigma)$ is a difference field.

Now let $U, V$ be varieties defined over $\mathcal{C}$, with $U \subset V \times V^{\sigma}$ such that $U$ projects generically over $V$ and $V^{\sigma}$. Let $W=U \times(\overline{0}) \subset \tau_{1}\left(V \times V^{\sigma}\right)$. Then, by 3.15 there is $a \in K$ such that $(a, D a, \sigma(a), \sigma(D a)) \in W$. Thus $D a=0$ and $(a, \sigma(a)) \in U$.

Remark 4.2 Clearly the fixed field of $\mathcal{C}$ is $\mathcal{C} \cap F i x \sigma$, and as the fixed field of a model of $A C F A$ is pseudofinite, $\mathcal{C} \cap$ Fix $\sigma$ is pseudofinite. Hence $\mathcal{C} \cap$ Fix $\sigma \prec F i x \sigma$ (in the language of fields).

Remark 4.3 Fix $\sigma$ is a differential field, however it is not differentially closed since it is not algebraically closed as a field. Clearly, it is also a difference field, thus

$$
\operatorname{acl}(F i x \sigma)=\operatorname{acl}_{D}(F i x \sigma)=(F i x \sigma)^{a l g} .
$$

Theorem $4.4\left((F i x \sigma)^{a l g}, D\right)$ is a model of DCF .

Proof:

Let $V, W$ be two irreducible affine varieties defined over $(F i x \sigma)^{\text {alg }}$ such that $W \subset \tau_{1}(V)$ and $W$ projects dominantly onto $V$. Let $k \in \mathbb{N}$ be such that both $V$ and $W$ are defined over Fix $\sigma^{k}$. Let $U=\{(x, x): x \in V\}$. Then $U \subset V \times V^{\sigma^{k}}=V \times V$.

Let $W^{\prime}=\{(y, y): y \in W\}$. Then $W^{\prime} \subset \tau_{1}(U)$. By $3.38\left(K, \sigma^{k}, D\right)$ is a model of $D C F A$; thus, applying 3.15 to $V, U$ and $W^{\prime}$ there is $a \in V(K)$ such that $\left(a, \sigma^{k}(a)\right) \in U$ and $\left(a, D a, \sigma^{k}(a), D\left(\sigma^{k}(a)\right)\right) \in W^{\prime}$. Thus $a=\sigma^{k}(a)$ and $(a, D a) \in W$. By 2.4, $\left((F i x \sigma)^{a l g}, D\right)$ is differentially closed.

Using (the proof of) 4.4, we can also axiomatize the theory of the structures $(F, D)$, where $F$ is the fixed field of a model of $D C F A$, as follows:

1. $F$ is a pseudo-finite field.

2. For every irreducible algebraic variety $V$ defined over $F$, if $W$ is an irreducible algebraic subvariety of $\tau_{1}(V)$ defined over $F$, such that the projection of $W$ onto $V$ is dominant, then there is $a \in V(F)$ such that $(a, D a) \in W$. 
For such a structure $(F, D)$ we can describe its completions, the types, the algebraic closure in the same way as we did for $D C F A$. For instance, if $F_{1}$ and $F_{2}$ are two models of this theory and $E$ is a common substructure, $F_{1} \equiv_{E} F_{2}$ if and only if there is an isomorphism $\varphi: E^{\text {alg }} \cap F_{1} \rightarrow E^{\text {alg }} \cap F_{2}$ which fixes $E$. If we add enough constants (for a pseudo finite field $F$ we add a set of constants $A \subset F$ such that $F A^{\text {alg }}=F^{\text {alg }}$ ), the generalized independence theorem will hold.

Pseudo-algebraically closed structures were studied by E. Hrushovski in a preprint of 91, to appear in the Ravello Proceedings. In [13] Pillay and Polkowska generalize Hrushovski's results and treat the differential case described above.

The field of constants of a model of $D C F$ as well as the fixed field of a model of $A C F A$ are stably embedded, that is any definable subset of $\mathcal{C}^{n}\left(F i x \sigma^{n}\right)$ is definable with parameters form $\mathcal{C}(F i x \sigma)$ in the language of fields. The same happens in DCFA for the field Fix $\sigma$ but not for the field $\mathcal{C}$.

Proposition $4.5(\mathcal{C}, \sigma)$ is not stably embedded.

Proof:

Let $a \in F i x \sigma \backslash \mathcal{C}$, then the set $\left\{x \in K: \exists y \sigma(y)=y \wedge D x=0 \wedge y^{2}=x+a\right\}$ is contained in $\mathcal{C}$ but it is not definable with parameters from $\mathcal{C}$.

Proposition 4.6 Let $A$ be a definable subset of $(\text { Fix } \sigma)^{n}$. Then $A$ is definable over Fix $\sigma$ in the language $\mathcal{L}_{D}$.

Proof :

Since $D C F A$ eliminates imaginaries, there is a canonical parameter $a$ for $A$. Since $A$ is fixed by $\sigma, a$ is fixed by $\sigma$, thus $A$ is $(F i x \sigma)$-definable. It is enough to show that there exist a countable subset $L$ of Fix $\sigma$ containing a such that every $\mathcal{L}_{D}$-automorphism of Fix $\sigma$ which fixes $L$ extends to an elementary map of some elementary extension of Fix $\sigma$.

Let $L$ be a countable elementary $\mathcal{L}_{D}$-substructure of Fix $\sigma$ containing $a$. In particular $L$ is a differential field, and $\operatorname{acl}(L)=L^{a l g}$.

Since $L \prec \mathcal{L}_{D}$ Fix $\sigma, L^{\text {alg }}$ and Fix $\sigma$ are linearly disjoint over $L$. If $L_{n}$ is the unique algebraic extension of $L$ of degree $n$, then $L_{n} F i x \sigma$ is the unique algebraic extension of Fix $\sigma$ of degree $n$; this implies that $(\text { Fix } \sigma)^{a l g}=L^{a l g}$ Fix $\sigma$.

Let $\tau$ be a $\mathcal{L}_{D^{-a u t o m o r p h i s m}}$ of Fix $\sigma$ over $L$. Then we can extend $\tau$ to a $\mathcal{L}_{D^{-}}$ automorphism $\bar{\tau}$ of $L^{\text {alg }}$ Fix $\sigma$ over $L^{\text {alg }}$. We have that $\bar{\tau}$ commutes with $\sigma$. Thus $\bar{\tau}$ is a $\mathcal{L}_{\sigma, D}$-automorphism of $\operatorname{acl}(\mathrm{Fix} \sigma)$. Then, by $3.25, \bar{\tau}$ is an elementary map.

Remark 4.7 Let $\left(L, L_{A}\right)$ be a pair of fields extending the pair of fields $($ Fix $\sigma, F i x \sigma \cap \mathcal{C})$ and which satisfies: $L$ is a regular extension of Fix $\sigma, L_{A}$ is a regular extension of $F i x \sigma \cap \mathcal{C}$, and Fix $\sigma$ and $L_{A}$ are linearly disjoint over Fix $\sigma \cap \mathcal{C}$.

Using the linear disjointness of $L_{A}$ and Fix $\sigma$ over Fix $\sigma \cap \mathcal{C}$ and 2.7, the derivation $D$ of Fix $\sigma$ extends to a derivation $D_{1}$ on $L$ which is 0 on $L_{A}$. Defining $\sigma$ to be the identity 
on $L$, the difference differential field $\left(L, D_{1}, i d\right)$ embeds (over Fix $\left.\sigma\right)$ into an elementary extension of $\mathcal{U}$. The following follows easily:

1. The pair $(F i x \sigma, F i x \sigma \cap \mathcal{C})$ is S.P.A.C., that is, if $a, b$ are tuples in some extension of Fix $\sigma$ such that Fix $\sigma \subset$ Fix $\sigma(a, b)$ and Fix $\sigma \cap \mathcal{C} \subset($ Fix $\sigma \cap \mathcal{C})(a)$ are regular, and Fix $\sigma$ is linearly disjoint from $(F i x \sigma \cap \mathcal{C})(a)$ over Fix $\sigma \cap \mathcal{C}$; then there is a zero $\left(a^{\prime}, b^{\prime}\right)$ of $I(a, b / F i x \sigma)$ such that $a^{\prime} \in F i x \sigma \cap \mathcal{C}$.

This notion was introduced by H. Lejeune (see [9]).

2. The theory of the structure Fix $\sigma$ is model complete in the following languages:

(a) The language of pairs of fields with enough constants to describe all algebraic extensions of Fix $\sigma$, and with $n$-ary relation symbols for all $n$ which interpretation in $(F i x \sigma, F i x \sigma \cap \mathcal{C})$ is that the elements $x_{1}, \cdots, x_{n}$ are $(F i x \sigma \cap \mathcal{C})$-linearly independent.

(b) The language of differential fields with enough constants to describe all algebraic extensions of Fix $\sigma$ (as in this language extensions are field extensions with an extension of the derivation this will automatically imply linear disjointness).

\section{References}

[1] Chatzidakis, Z.; Hrushovski, E. (1999) "Model theory of difference fields", Transactions of the American Mathematical Society 351(8): 2997-3071.

[2] Chatzidakis, Z.; Pillay, A. (1998) "Generic structures and simple theories", Annals of Pure and Applied Logic 95(1-3): 71-92.

[3] Cohn, R.M. (1965) Difference Algebra. Interscience Publishers John Wiley \& Sons, New York-London-Sydney.

[4] Cohn, R.M. (1969) "Systems of ideals", Canad. J. Math. 21: 783-807.

[5] Cohn, R.M. (1970) "A difference-differential basis theorem", Canad. J. Math. 22: $1224-1237$.

[6] Kim, B.; Pillay A. (1997) "Simple theories", Annals of Pure Applied Logic, 88(2$3): 149-164$.

[7] Kolchin, E.R. (1973) Differential Algebra and Algebraic Groups. Pure and Applied Mathematics, Vol. 54, Academic Press, New York.

[8] Lang, S. (1965) Algebra. Addison-Wesley Publishing Co., Inc., Reading, Massachusets.

[9] Lejeune, H. (1995) Paires de Corps P.A.C. Parfaits, Paires de Corps Pseudofinis. Thèse de Doctorat, Université de Paris 7, Paris. 
[10] Marker, D.; Messmer, M.; Pillay, A. (1996) Model Theory of Fields, volume 5 of Lecture Notes in Logic. Springer-Verlag, Berlin.

[11] Marker, D. (2000) "Model theory of differential fields", in: Haskell, D. Pillay, A. Steinhorn, C. (Eds.) Model Theory, Algebra, and Geometry, volume 39 of Math. Sci. Res. Inst. Publ., Cambridge Univ. Press, Cambridge: pages 53-63.

[12] Pierce, D.; Pillay, A. (1998) "A note on the axioms for differentially closed fields of characteristic zero", J. Algebra 204(1): 108-115.

[13] Pillay, A.; Polkowska, D. (2004) "On PAC and bounded substructures of a stable structure", Preprint, University of Illinois at Urbana-Champaign, Illinois.

[14] Poizat, B. (1985) Cours de Théorie des Modèles. Une introduction à la logique mathématique contemporaine. Bruno Poizat, Lyon.

[15] van den Dries, L.; Schmidt, K. (1984) "Bounds in the theory of polynomial rings over fields. A nonstandard approach", Invent. Math. 76(1): 77-91.

[16] Wood, C. (1998) "Differentially closed fields", in: E. Bouscaren (Ed.) Model Theory and Algebraic Geometry, volume 1696 of Lecture Notes in Math., Springer, Berlin: pages 129-141. 